\title{
A Matter of Context: How an Understanding of Redox Homeostasis Informs the Consideration of Pro-oxidant Strategies to Target Tuberculosis, HIV, and Cancer Metastasis
}

\section{David J Waters* and Emily C Chiang}

Center for Exceptional Longevity Studies, Gerald P. Murphy Cancer Foundation, USA

*Corresponding Author: David J. Waters, Center for Exceptional Longevity Studies, Gerald P. Murphy Cancer Foundation, USA.

Received: September 03, 2019; Published: September 13, 2019

Keywords: Antioxidant; Disease; $\mathrm{H}_{2} \mathrm{O}_{2}$ Signaling; Oxidative Stress; Physiological Context; Pro-Oxidant Strategies; Reactive Oxygen Species (ROS); Redox Homeostasis

For decades, conventional thinking has championed the idea that oxidative stress - the internal and external factors that give rise to oxidative damage to cellular proteins, membranes, and DNA - was "bad", serving as the rationale for using dietary supplementation with antioxidants as a strategy to promote health and fight disease. Yet, even today, the precise role of reactive oxygen species (ROS) in most human diseases remains mostly unknown [1,2]. In some instances, elevated markers of oxidative damage may merely correlate with pathology, rather than be causative. Antioxidant interventions may lower oxidative damage, but still have no significant impact on disease outcomes. Methodological shortcomings encountered in the measurement of ROS production and oxidative damages need to be meticulously addressed so that sound conclusions can be drawn from the burgeoning number of clinical and mechanistic studies [1].

A deepening of our understanding of the role of ROS in human disease will require drawing our attention to how ROS operate within a vast number of distinctive physiological contexts. What is needed are conceptual frameworks that will enable us to move beyond generalized, monolithic statements, such as "Oxidative stress is bad", because these statements may distort or conceal the beneficial role that ROS actually plays in health and disease. The two terms introduced by Helmut Sies [3] to distinguish between conditions of physiological reduction-oxidation (redox) signaling (oxidative eustress) and conditions of disrupted redox signaling and oxidative damage (oxidative distress) serve as an example of such an explanatory framework. What emerges from this kind of dual thinking is an appreciation for the potential importance of redox- dependent targets that can substantially impact cellular function.

The concept of redox homeostasis

Growing evidence indicates the essential role that ROS plays in regulating a wide range of biological phenomena [2,4]. Reactive oxygen species - once clearly ear-marked as the villainous contributor to cell damages - are now being seen in a different light. The previously satisfying view of chaotic, dysregulated ROS production leading to the damage of everything in its wake has given way to a richer picture of biological complexity. Tightly-regulated ROS production (particularly hydrogen peroxide, $\mathrm{H}_{2} \mathrm{O}_{2}$ ) along with tightly-regulated systems dedicated to $\mathrm{H}_{2} \mathrm{O}_{2}$ removal contribute to redox homeostasis. To maintain intracellular redox state within these workable limits, cells are dynamically equipped to respond or adapt to changes in their redox state, including critical spatiotemporal variations in the concentration of oxidants and reductants.

Some of the key players contributing to redox homeostasis are depicted in figure 1. ROS are generated by the electron transport chain of mitochondria as a by-product of cellular respiration and ATP production. ROS are also produced by a group of professional ROS-generating enzymes called NADPH oxidases (NOX) [3,5]. Tissue NOX orchestrates the availability of $\mathrm{H}_{2} \mathrm{O}_{2}$ in response to diverse physiological stimuli. For example, following epidermal growth factor (EGF) ligand-receptor binding, $\mathrm{H}_{2} \mathrm{O}_{2}$ production leads to oxidation of protein tyrosine phosphatases resulting in their inactivation. This inactivation temporarily shifts the signaling balance toward unopposed tyrosine kinase activity, leading to the phosphorylation (activation) of downstream targets responsible for EGF action. 


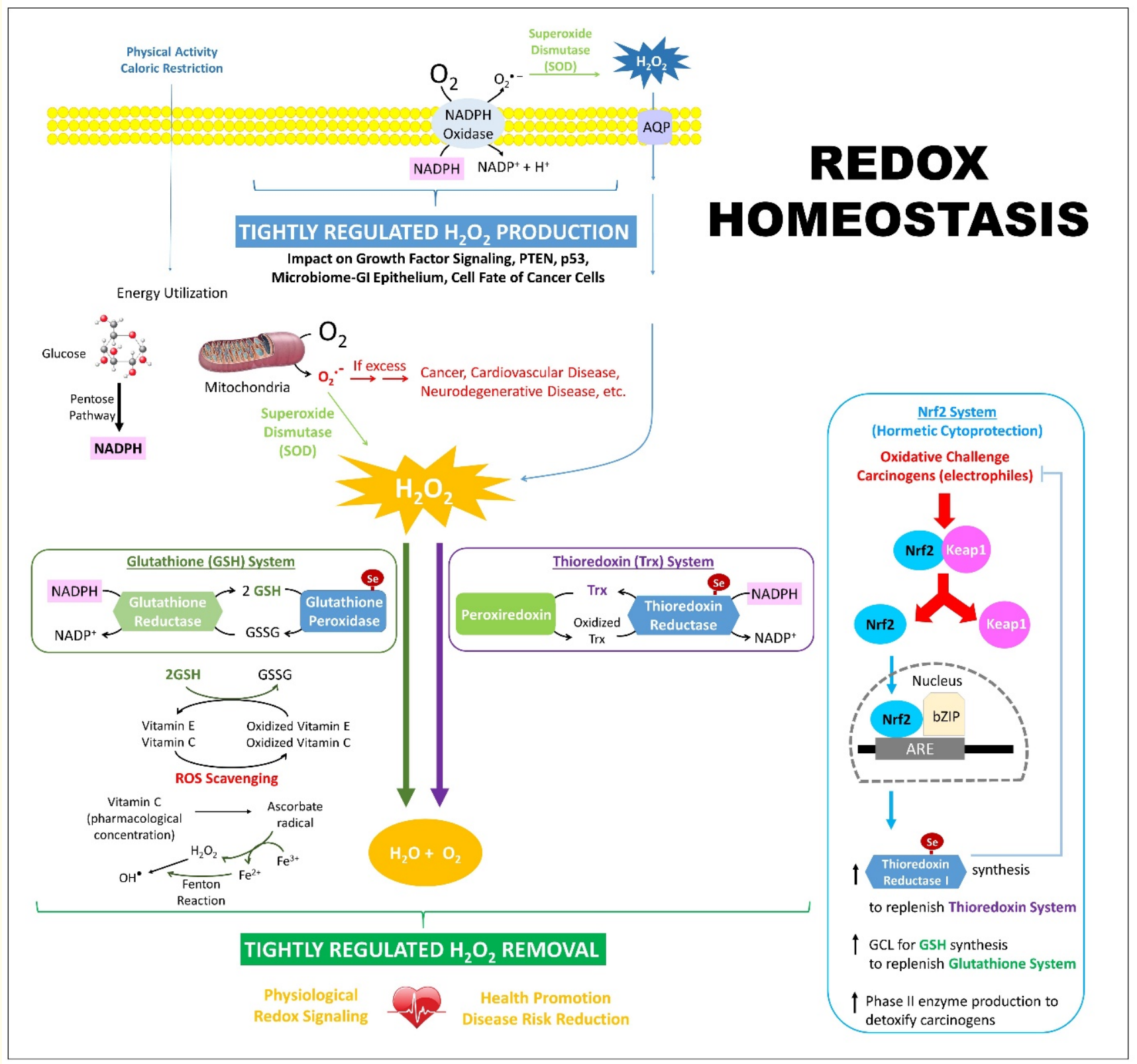

Figure 1: The essential role of the glutathione system, thioredoxin system, and the Nrf2 pathway in the regulation of redox homeostasis. ARE, antioxidant response element; AQP, aquaporin; bZIP, basic leucine zipper; $\mathrm{Fe}^{3+}$, ferric iron; $\mathrm{Fe}^{2+}$, ferrous iron; GCL, glutamatecysteine ligase; GSH, glutathione; GSSG, glutathione disulfide; $\mathrm{H}+$, hydrogen; $\mathrm{H}_{2} \mathrm{O}_{2}$, hydrogen peroxide; Keap1, Kelch-like ECH-associated protein 1; NADP, nicotinamide adenine dinucleotide phosphate; NADPH, nicotinamide adenine dinucleotide phosphate hydrogen; Nrf2, nuclear factor erythroid 2-related factor 2; 02, oxygen; $02 \bullet$; superoxide anion; $\mathrm{OH} \bullet$, hydroxyl radical; ROS, reactive oxygen species; Se, selenium; Trx, thioredoxin 
A Matter of Context: How an Understanding of Redox Homeostasis Informs the Consideration of Pro-oxidant Strategies to Target Tuberculosis, HIV, and Cancer Metastasis

Reactive oxygen species differ in their capacity to damage macromolecules and to serve as signaling moieties. Superoxide anion generated by mitochondria and by NOX is readily converted by superoxide dismutase (SOD) to $\mathrm{H}_{2} \mathrm{O}_{2}$. By removing superoxide anion, the cell is protected from the generation of hydroxyl radical $(\mathrm{OH})$, a highly reactive, toxic species for which cells have evolved no enzymatic systems to eliminate. Pharmacologic concentrations of the ROS scavenger vitamin $\mathrm{C}$ can exert pro-oxidant effects, serving as a prodrug for the formation of $\mathrm{H}_{2} \mathrm{O}_{2}$ in the extracellular space [6]. $\mathrm{H}_{2} \mathrm{O}_{2}$ can diffuse into cells, and in the presence of ferrous ions $\left(\mathrm{Fe}^{2+}\right)$, react to form hydroxyl radical via the Fenton reaction. Based on its stability and intracellular concentration gradient, $\mathrm{H}_{2} \mathrm{O}_{2}$ seems to be best suited among the various ROS for mediating redox reactions under physiologic conditions [3].

Not unexpectedly then, ROS production whether by NOX or by mitochondria, is funneled toward $\mathrm{H}_{2} \mathrm{O}_{2}$, for which two major systems have evolved for its removal: the glutathione (GSH) system and the thioredoxin (Trx) system. Both systems rely upon selenium (Se)-dependent enzymes [7]: glutathione peroxidase (GPx), and thioredoxin reductase (TrxR) which regenerates (reduces) thioredoxin necessary for the recycling of peroxiredoxins, which are major handlers of the large amounts of $\mathrm{H}_{2} \mathrm{O}_{2}$ produced in the brain [8]. Redox homeostasis is tightly linked to energy metabolism because NADPH, produced from the shuttling of glucose through the pentose pathway, supports both the production of $\mathrm{H}_{2} \mathrm{O}_{2}$ by NOX and removal of $\mathrm{H}_{2} \mathrm{O}_{2}$ by the glutathione and thioredoxin systems.

A third pathway involves the master regulator known as nuclear factor erythroid 2-related factor 2 (Nrf2), which integrates environmental challenges with redox homeostasis, sometimes referred to as hormetic cytoprotection [9]. In response to carcinogens or oxidative challenge by other electrophiles, Nrf2 is translocated into the nucleus where it binds to antioxidant response element (ARE), driving the transcription of key players: glutamatecysteine ligase (GCL), the enzyme that catalyzes the rate-limiting step in the synthesis of glutathione [10]; thioredoxin reductase-1 (TrxR1), which is one of the factors that returns Nrf2 to a resting state, readied for subsequent challenge [9]; and phase II enzymes, such as glutathione S-transferases which detoxify carcinogens. It is instructive to note that the Nrf2 pathway is highly regulated by Keap1, which is itself a redox-sensitive protein. Under reducing conditions, Keap1 promotes the proteosomal degradation of Nrf2 in the cytosol, thereby preventing Nrf2 transcription [11].
The picture that can be painted is a cellular display capable of delivering rapid, reversible responses mediated by the action of $\mathrm{H}_{2} \mathrm{O}_{2}$ on the Cys, SeCys and Met residues of redox-sensitive proteins, analogous to the critical switching role that phosphorylation - dephosphorylation of Ser and Thr residues plays in tissue homeostasis and normal physiology. It is not surprising then that diverse physiological processes, ranging from sleep and circadian rhythms, to autophagy and the adaptation of skeletal muscle to exercise, are redox sensitive [12-15].

If redox-sensitive processes are an integral part of cell-sustaining responses to factors that challenge homeostasis, might certain pathologies represent a breakdown or inadequacy of ROS-mediated action, rather than the all-too-often assumed consequences of ROS-induced damage? Such questioning, once considered heretical, is now leading investigators to explore the possible benefit of pro-oxidant strategies against human diseases. Let us examine some of the compelling, albeit preliminary, findings.

\section{Tuberculosis (TB)}

Tuberculosis is a leading cause of mortality world-wide [16]. The disease occurs in susceptible persons exposed to a bacterium (most commonly Mycobacterium tuberculosis, Mtb) leading to pathologic damage of lung and other tissues. As infection proceeds, $M t b$ replicates within host macrophages. In response to the invading pathogen, tissue macrophages initiate a respiratory burst, producing high levels of ROS and nitric oxide in an attempt to eradicate the bacteria [17]. Not surprisingly, $M t b$ has co-evolved extensive systems to maintain its own intracellular redox balance, enabling the pathogen to survive and replicate within hostile host macrophages [18]. Prolonged (6 months) drug treatment can be effective, but resistance to first-line antibiotic treatment (e.g., isoniazid, rifampin) has increasingly become a threat to global health [19].

But treatment failure in TB is not limited to the acquisition of multiple drug resistance. $M t b$ can evade drug killing by assuming a state of metabolic dormancy [20]. The dormant state of these "persister cells" is characterized by low cellular respiration, low ROS production, and resistance to bactericidal antibiotics. Could the manipulation of cellular respiration [21] in drug-insensitive persistent $M t b$ serve as a pro-oxidant strategy that would lead to eradication of dormant bacteria? The rationale for pursuing this kind of approach is strengthened further by the discovery that although $M t b$ is endowed with robust defenses against exogenous oxidative assaults by host macrophages, $M t b$ is highly sensitive to perturbations in 
A Matter of Context: How an Understanding of Redox Homeostasis Informs the Consideration of Pro-oxidant Strategies to Target Tuberculosis, HIV, and Cancer Metastasis

endogenous ROS levels [22].

Investigators are now studying closely the redox context that supports $M t b$ persistence. Recent in vitro studies by Vilchèze., et al. [23] have shown that administering small thiols (e.g. cysteine) to induce higher $M t b$ respiration can convert $M t b$ persisters to metabolically active bacteria. This metabolic conversion led to increased concentration of endogenous ROS and sterilization of cultures with treatment using the first-line anti-Mtb drug isoniazid. The investigators attributed the Mtb killing to endogenous ROSinduced DNA damage.

Further evidence for the potential benefits of such pro-oxidant strategies to enhance the efficacy of existing anti-Mtb chemotherapy comes from experiments in which the bactericidal effects of pharmacologic dosing with vitamin $\mathrm{C}$ on $M t b$ were accompanied by upregulation of $M t b$ ROS production [24,25]. In separate experiments [26], the combination of vitamin $C$ and isoniazid induced more rapid sterilization of $M t b$ cultures than using isoniazid alone. Moreover, intraperitoneal vitamin $C$ treatment potentiated the ability of isoniazid and rifampin to decrease lung burden of $M t b$ in mice following aerosol infection.

HIV

The human immunodeficiency virus type 1 (HIV-1) is a lentivirus that causes selective immune cell destruction, which can lead to the life-threatening immunodeficiency syndrome known as AIDS [27]. Remarkable advances in the use of combination antiretroviral therapy (referred to as cART or HAART) can effectively suppress HIV viral load, saving lives, but cannot completely eliminate transcriptionally inactive (latent) viral reservoirs. Among the current challenges facing interventionalists is how to eradicate these latent HIV reservoirs [28] and how to minimize the adverse consequences of chronic immune activation, such as the elevated risk for chronic, non-communicable diseases that occurs among HIV treatment responders [29].

Redox alterations associated with HIV infection have been extensively catalogued, with one review concluding that "HIV infection leads to pronounced oxidative stress" [30]. Recently, investigators are turning to new classes of anti-HIV compounds that target the patient's redox-regulating enzyme thioredoxin reductase-1 (TrxR1). But the aim of this approach is not augmentation of TrxR1 activity, but rather inhibition.
The rationale behind this partially unexpected approach stems from the observation that the entry of HIV-1 into host cells is a redox-sensitive event [31]. Specifically, cellular TrxR1 plays an essential role in HIV-1 entry into CD4+ T lymphocytes. The Trx-1 - TrxR1 system is responsible for reduction of disulfide bridges in the gp120 viral envelope protein of HIV. Failure of this reducing activity results in conformational changes in gp120 that prohibit fusion of host cell membranes and viral membranes. The gold-containing compound auranofin and organotellurium compounds are among the agents that have been shown to block HIV entry using this prooxidative TrxR1 - inhibiting approach [32].

Since central and transitional memory $\mathrm{T}$ cells serve as a major reservoir for transcriptionally inactive virus in cART treated patients, approaches are being pursued to selectively remove these memory lymphocyte subsets. Again, the pro-oxidant auranofin, a potent inhibitor of thioredoxin reductases, was shown to have beneficial effects, preferentially triggering apoptosis in these memory subsets, presumably by exploiting the already lower baseline antioxidant defenses of these cells compared to naïve $\mathrm{T}$ lymphocytes [33]. More recently, the efficacy and safety of auranofin - buthionine sulfoximine (BSO) combinations have been demonstrated in a simian model of HIV [34]. The rationale for using this dual targeting combination is that BSO, a selective inhibitor of glutathione synthesis, can act in concert with auranofin as a pro-oxidant by depleting intracellular glutathione [35]. The suggestion that the establishment and persistence of HIV reservoirs may be redox sensitive and therefore susceptible to pro-oxidant targeting requires further investigation.

\section{Cancer metastasis}

Cancer metastasis is the multi-step process by which cancer cells escape the primary tumor to establish deposits in distant locations within the body. Metastasis, not the local effects of primary tumor growth, accounts for the vast majority of cancer-attributable deaths. Metastasis is a highly inefficient process - few tumor cells gaining access to the systemic circulation are capable of successfully establishing a tumor metastasis in the foreign soil of a distant target organ [36].

The use of antioxidants to reduce the risk of cancer development has been proposed and large-scale prevention trials have been conducted with few favorable results [37,38]. However, a study published in Nature by Piskounova., et al. [39] has provided a closer appraisal of the redox context of cancer cells. The results of 
A Matter of Context: How an Understanding of Redox Homeostasis Informs the Consideration of Pro-oxidant Strategies to Target Tuberculosis, HIV, and Cancer Metastasis

this study in mice are intriguing: ROS may limit cancer metastasis. Exposure to the antioxidant, $\mathrm{N}$-acetylcysteine (NAC), had no effect on primary tumor growth but increased the number of circulating cancer cells and increased the formation of distant colonies. The experiments revealed that metastasizing tumor cells underwent reversible metabolic changes during metastasis that increased their capacity to withstand oxidative stress. The finding that cellular oxidative stress limits metastasis in vivo opens the door to the development of pro-oxidant drugs or inhibitors of antioxidant responses to target lethal cancer progression.

In separate work using mouse xenograft models, antioxidant antidiabetic agents increased cancer metastasis, without affecting the incidence of tumor formation [40]. Consistent with the work cited above, those agents that activated Nrf2 - one of the prime pathways used to upregulate antioxidant defenses - had the most potent metastasis-promoting effect. These results were subsequently confirmed by experiments showing that targeted genetic overexpression or pharmacological upregulation of Nrf2 increased the ability of cancer cells to migrate and metastasize [40]. Taken together, the results suggest that the preferred choice of antioxidant versus pro-oxidant strategies may differ in cancer chemopreventive versus cancer treatment settings [41].

Synthesis

A proposition is presented that a deeper appreciation for the complexity of redox homeostasis and how it relates to biological regulation in different contexts might contribute significantly to our search for health-promoting and disease-fighting interventions. The proposition urges movement away from overgeneralized, stagnant statements about the purely harmful aspects of ROS that may cut us off from new avenues of discovery. Context matters and the words we use to describe the particular contexts contributing to biological phenomena are our critical starting points - guiding today's clinical practice, fueling tomorrow's inquiry. The growing body of evidence that the intentional production of ROS plays a critical role in cell signaling and tissue homeostasis, encourages us to grow our receptivity to the idea that strategies that exert pro-oxidant effects may actually be beneficial to the host under certain circumstances. This pregnant possibility is illustrated here by ongoing research in the fields of tuberculosis, HIV, and cancer metastasis. Finally, the proposition carries no insinuation of finishedness, but rather a call for seeing these new starting points glittering amidst old ways. And though much uncertainty re- mains, we should be communicating with elevated confidence our need for nuanced approaches, the kind of approaches that incite attention to the primacy of context as we work to define more completely the role of ROS in health and disease.

\section{Bibliography}

1. Murphy MP., et al. "Unraveling the biological roles of reactive oxygen species". Cell Metabolism 13 (2011): 361-366.

2. Holmström KM and Finkel T. "Cellular mechanisms and physiological consequences of redox-dependent signaling”. Molecular Cell Biology 15 (2014): 411-421.

3. Sies H. "Hydrogen peroxide as a central redox signaling molecule in physiological oxidative stress: Oxidative eustress". Redox Biology 11 (2017): 613-619.

4. Ursini F., et al. "Redox homeostasis: The golden mean of healthy living". Redox Biology 8 (2016): 205-215.

5. Lambeth JD and Neish AS. "Nox enzymes and new thinking on reactive oxygen: A double-edged sword revisited". Annual Review of Pathology 9 (2014): 119-145.

6. Chen Q., et al. "Ascorbate in pharmacologic concentrations selectively generates ascorbate radical and hydrogen peroxide in extracellular fluid in vivo". Proceedings of the National Academy of Sciences of the United States of America 104 (2007): 8749-8754.

7. Lei XG., et al. "Paradoxical roles of antioxidant enzymes: Basic mechanisms and health implications". Physiological Reviews 96 (2016): 307364.

8. Bell KFS and Hardingham GE. "CNS peroxiredoxins and their regulation in health and disease". Antioxidants and Redox Signaling 14 (2011): 1467-1477.

9. Brigelius-Flohé $\mathrm{R}$ and Flohé L. "Basic principles and emerging concepts in the redox control of transcription factors". Antioxidants and Redox Signaling 15 (2011): 2335-2381.

10. Lu SC. "Glutathione synthesis". Biochimica et Biophysica Acta 1830 (2013): 3143-3153.

11. McMahon M., et al. "Keap1-dependent proteasomal degradation of transcription factor Nrf2 contributes to the negative regulation of antioxidant response element-driven gene expression". Journal of Biological Chemistry 278 (2003): 21592-21600.

12. Wu JJ., et al. "Mitochondrial dysfunction and oxidative stress mediate the physiological impairment induced by the disruption of autophagy". Aging 1 (2009): 425-437.

13. Merry TL and Ristow M. "Do antioxidant supplements interfere with skeletal muscle adaptation to exercise training". Journal of Physiology 594 (2016): 5135-5147. 
A Matter of Context: How an Understanding of Redox Homeostasis Informs the Consideration of Pro-oxidant Strategies to Target Tuberculosis, HIV, and Cancer Metastasis

14. Kempf A., et al. "A potassium channel $\beta$-subunit couples mitochondrial electron transport to sleep". Nature 568 (2019): 230-234.

15. Edgar RS., et al. "Peroxiredoxins are conserved markers of circadian rhythms". Nature 485 (2012): 459-464.

16. Floyd K., et al. "The global tuberculosis epidemic and progress in care, prevention, and research: An overview in year 3 of the End TB era". Lancet Respiratory Medicine 6 (2018): 299-314.

17. Shastri MD., et al. "Role of oxidative stress in the pathology and management of human tuberculosis". Oxidative Medicine and Cellular longevity 2018 (2018): 7695364.

18. Kumar, A., et al. "Redox homeostasis in mycobacteria: The key to tuberculosis control". Expert Reviews in Molecular Medicine 13 (2011): e39.

19. Raviglione M and Sulis, G. "Tuberculosis 2015: Burden, challenges and strategy for control and elimination". Infectious Disease Reports 8 (2016): 33-37.

20. Wakamoto Y., et al. "Dynamic persistence of antibiotic-stressed mycobacteria”. Science 339 (2013): 91-95.

21. Lobritz MA., et al. "Antibiotic efficacy is linked to bacterial cellular respiration". Proceedings of the National Academy of Sciences of the United States of America 112 (2015): 8173-8180.

22. Tyagi P., et al. "Mycobacterium tuberculosis has diminished capacity to counteract redox stress induced by elevated levels of endogenous superoxide". Free Radical Biology and Medicine 84 (2015): 344-354.

23. Vilchèze C., et al. "Enhanced respiration prevents drug tolerance and drug resistance in Mycobacterium tuberculosis". Proceedings of the National Academy of Sciences of the United States of America 114 (2017): 4495-4500.

24. Vilchèze C., et al. "Mycobacterium tuberculosis is extraordinarily sensitive to killing by a vitamin C-induced Fenton reaction". Nature Communications 4 (2013): 1881.

25. Pei Z., et al. "Pharmacologic ascorbate as a pro-drug for hydrogen peroxide release to kill mycobacteria". Biomedicine and Pharmacotherapy 109 (2019): 2119-2127.

26. Vilchèze C., et al. "Vitamin C potentiates the killing of Mycobacterium tuberculosis by the first-line tuberculosis drugs isoniazid and rifampin in mice". Antimicrobial Agents Chemotherapy 62 (2018): e02165-17.

27. Ghosn J., et al. "HIV”. Lancet 392 (2018): 685-697.

28. Wong JK and Yukl SA. "Tissue reservoirs of HIV". Current Opinion in HIV and AIDS 11 (2016): 362-370.

29. Guaraldi G., et al. "Premature age-related comorbidities among HIVinfected persons compared with the general population". Clinical Infectious Diseases 53 (2011): 1120-1126.
30. Ivanov AV., et al. "Oxidative stress during HIV infection: Mechanisms and consequences". Oxidative Medicine and Cellular Longevity 2016 (2016): 8910396

31. Ryser HJ., et al. "Inhibition of human immunodeficiency virus infection by agents that interfere with thiol-disulfide interchange upon virusreceptor interaction". Proceedings of the National Academy of Sciences of the United States of America 91 (1994): 4559-4563.

32. Reiser K., et al. "The cellular thioredoxin-1/thioredoxin reductase-1 driven oxidoreduction represents a chemotherapeutic target for HIV-1 entry inhibition". PLoS ONE 11 (2016): e0147773.

33. Chirullo B., et al. "A candidate anti-HIV reservoir compound, auranofin, exerts a selective "anti-memory" effect by exploiting the baseline oxidative status of lymphocytes". Cell Death and Disease 4 (2013): e944.

34. Shytaj IL., et al. "Two-year follow-up of macaques developing intermittent control of the human immunodeficiency virus homolog simian immunodeficiency virus SIVmac251 in the chronic phase of infection". Journal of Virology 89 (2015): 7521-7535.

35. Benhar M., et al. "Dual targeting of the thioredoxin and glutathione systems in cancer and HIV". Journal of Clinical Investigation 126 (2016): 1630-1639.

36. Massagué J and Obenauf AC. "Metastatic colonization by circulating tumor cells". Nature 529 (2016): 298-306.

37. Waters DJ and Chiang EC. "Five threads: How U-shaped thinking weaves together dogs, men, selenium, and prostate cancer risk". Free Radical Biology and Medicine 127 (2018): 36-45.

38. Waters DJ and Chiang EC. "Prostate cancer risk reduction achievable through selenium supplementation: Looking back to move forward". Acta Scientific Nutritional Health 3 (2019): 45-50.

39. Piskounova E., et al. "Oxidative stress inhibits distant metastasis by human melanoma cells”. Nature 527 (2015): 186-191.

40. Wang H., et al. "NRF2 activation by antioxidant antidiabetic agents accelerates tumor metastasis". Science Translational Medicine 8 (2016): $334 \mathrm{ra} 51$.

41. Sporn MB and Liby KT. "NRF2 and cancer: The good, the bad, and the importance of context”. Nature Reviews Cancer 12 (2012): 564-571.

\section{Volume 3 Issue 10 October 2019 (c) All rights are reserved by David J Waters and Emily C Chiang.}

